\title{
COMPREENSÃO DO CONCEITO DE TAXA DE VARIAÇÃO POR PROFESSORES EM FORMAÇÃO CONTINUADA
}

\section{COMPREHENSION OF THE VARIATION RATE CONCEPT BY TEACHERS IN CONTINUED TRAINING}

\author{
Vanilde Bisognin ${ }^{1}$ \\ Eleni Bisognin ${ }^{2}$
}

\begin{abstract}
Resumo: Neste trabalho, são apresentados resultados de uma pesquisa que tem como objetivo analisar como professores em formação continuada, participantes da disciplina de Fundamentos de Cálculo Diferencial de um curso de Mestrado em Ensino de Matemática, interpretam e relacionam as informações explicitadas pelas diferentes representações do conceito de taxa de variação. Para tanto foi aplicada uma sequência de atividades sobre as diferentes representações do conceito de taxa de variação e as respostas das questões foram analisadas e categorizadas. Os resultados apontam que, apesar de terem trabalhado o conceito de taxa de variação em seus cursos de licenciatura em Matemática, alguns professores ainda apresentam dificuldades para compreender esse conceito e relacionar suas diferentes representações. Pela análise dos dados, considera-se que as atividades propostas proporcionaram a evolução da ideia intuitiva de taxa de variação e favoreceram a compreensão desse conceito pelos participantes da pesquisa.
\end{abstract}

Palavras-chave: Taxa de variação; Formação de professores; Imagem de conceito; Definição de conceito.

\begin{abstract}
In this work, results of a research are presented that aim to analyze how teachers in continuous formation, participants in the discipline of Fundamentals of Differential and Integral Calculus of a Master course in Mathematics Teaching, interpret and relate the information explained by the different representations of the concept of rate of variation. For this, a sequence of activities was applied on the different representations of the concept of rate of variation and the answers of the questions were analyzed and categorized. The results show that, although they have worked on the concept of rate of variation in their undergraduate courses in Mathematics, some teachers still find it difficult to understand this concept and to relate their different representations. By analyzing the data, it is considered that the proposed activities provided the evolution of the intuitive idea of rate of variation and favored the understanding of this concept by the research participants.
\end{abstract}

Keywords: Rate of variation; Teacher training; Concept image; Definition of concept.

\section{Introdução}

Os conteúdos da disciplina de Cálculo Diferencial e Integral que constam das matrizes curriculares dos cursos de graduação em Matemática, tanto na modalidade Bacharelado quanto Licenciatura, incluem a noção de "taxa de variação" como um dos

\footnotetext{
${ }^{1}$ Doutora em Matemática pela Universidade Federal do Rio de Janeiro (UFRJ). Professora Titular da Universidade Franciscana e participante do Curso de licenciatura em Matemática e do Programa de Pósgraduação em Ensino de Ciências e de Matemática, Santa Maria, Rio Grande do Sul, Brasil. E-mail: vanildebisognin@gmail.com

${ }^{2}$ Doutora em Matemática pela Universidade Federal do Rio de Janeiro (UFRJ). Professora do Programa de Pós-graduação em Ensino de Ciências e de Matemática da Universidade Franciscana (UFN), Santa Maria, Rio Grande do Sul, Brasil. E-mail: eleni.bisognin@gmail.com
} 
conceitos de fundamental importância para resolução de problemas, ou modelos oriundos da própria Matemática ou de outras áreas do conhecimento.

Analisando os documentos oficiais que orientam os currículos dos cursos de graduação para formação de professores de Matemática, Parecer CNE/CES 1302/2001 BRASIL (2001), indicam o conteúdo de taxa de variação e derivada, e recomendam que sejam abordados os aspectos analíticos, gráficos e numéricos, bem como suas aplicações.

Os conceitos referentes ao Cálculo Diferencial e Integral e as dificuldades relativas ao ensino e aprendizagem de tópicos de Matemática no Ensino Superior têm sido tema de pesquisas na área de Educação Matemática. Dentre elas, podem-se destacar os trabalhos de Vinner (1989), Tall (1994), Meyer e Igliori (2003), Meyer (2003), Almeida e Igliori (2013), Pinto e Cunha (2014), Vrancken e Engler (2014), bem como as investigações que fazem parte dos capítulos dos livros organizados por Frota e Nasser (2009) e Frota, Bianchini e Carvalho (2013). Os resultados apontam que uma das dificuldades de aprendizagem pelos estudantes estão relacionadas às noções de limite e derivada de funções reais. As mesmas pesquisas indicamtambém, que essas dificuldades estão relacionadas,em grande parte,com o modo como estes conceitos são abordados. Elas apontam que os estudantes de Cálculo têm melhor desempenho quando realizam atividades em que predominam questões que enfocam os aspectos operatórios e técnicos.

Na presente pesquisa, em se tratando do conceito de Taxa de Variação, a maioria dos professores participantes do trabalho informou que, em seus cursos de graduação, o conceito foi introduzido sem estar relacionado a uma situação concreta, sendo explicado diretamente a partir de sua definição. Isto é, não foram exploradas as múltiplas representações do conceito.

Referente ao conceito de derivada, Tall (1994), desenvolveu uma pesquisa cujo resultado aponta que estudantes universitários apresentam dificuldades em fazer a conexão entre as diferentes representações do conceito, principalmente entre as representações analítica e gráfica. $\mathrm{O}$ autor ressalta, ainda, que este fato pode estar ligado ao tipo de trabalho de sala de aula, em que prevalecem os aspectos técnicos.

As dificuldades apontadas nos resultados das investigações referenciadas motivaram a presente pesquisa que teve as seguintes questões norteadoras: como os professores participantes de um curso de mestrado profissional em Ensino de Matemática compreendem o conceito de Taxa de Variação? Como esses professores interpretam e relacionam as informações explicitadas pelas diferentes representações do conceito de taxa de variação? 
A pesquisa foi realizada com professores em formação continuada, participantes de um curso de mestrado em Ensino de Matemática, tendo como aporte teórico as ideias de "imagem de conceito" e "definição de conceito", conforme Tall e Vinner (1981), bem como as conceituações de "representações", de Tall (1992; 1994).

\section{Fundamentação teórica}

O desenvolvimento cognitivo de uma pessoa, associado a um conceito matemático, advém do acúmulo de diferentes experiências, relacionadas ao conceito. De acordo com Tall e Vinner (1981), um conceito não deve ser inicialmente trabalhado a partir de sua definição formal. Antes disso é necessário construir uma gama de ideias associadas ao conceito, isto é, é necessário criar diferentes imagens relacionadas ao conceito.

Para tentar compreender como se processa a aquisição de conhecimentos matemáticos, Tall e Vinner (1981) propuseram as ideias de "imagem de conceito" e "definição de conceito".

Para esses autores, a "imagem de conceito"

[...] descreve a estrutura cognitiva que está associada ao conceito que inclui todas as figuras mentais e propriedades associadas. Ela é desenvolvida ao longo dos anos, através de experiências de todos os tipos, mudando enquanto o indivíduo encontra novos estímulos e amadurece (TALL; VINNER, 1981, p.2).

Os autores expõem que, no trabalho de sala de aula, muitas vezes, há uma desarmonia entre a linguagem usada pelo professor e a linguagem matemática. Essa dissonância está relacionada com a complexidade do cérebro humano, que funciona de uma forma diferente da lógica matemática. Cada indivíduo pensa de um modo e, na sala de aula, uma ideia pode ser entendida por alguns alunos e não compreendida por outros.

$\mathrm{Na}$ construção de conceitos matemáticos, as diferentes representações desempenham um papel fundamental no sentido de facilitar a compreensão e contribuir significativamente para a construção de imagens conceituais que levem, de fato, à compreensão do conceito.

De acordo com Tall (2004), a trajetória de vida de cada um é uma experiência individual e, às vezes, ocorrem obstáculos os quais exigem que ideias anteriores devam ser reconsideradas ou reconstruídas. Os indivíduos são diferentes e lidam de modo diferente perante cada obstáculo o que leva a uma variedade de acontecimentos pessoais 
em que, alguns permitem que o indivíduo progrida de modo significativo e outros, levam a concepções errôneas.

No caminho da aprendizagem de um novo conceito, ocorrem avanços e dificuldades que, muitas vezes, exigem experiências anteriores para serem superadas. Cada sujeito tenta superar essas dificuldades de modo distinto e de acordo com suas experiências prévias. Essas experiências prévias são os "já-encontrados" que é um termo utilizado por Tall $(2004 ; 2013)$ e que estão presentes na imagem de conceito do sujeito. O autor utiliza o termo "a-encontrar" para designar uma experiência presente e que afeta a memória do conhecimento anterior.

Para Lima (2007), os termos “já-encontrados” e "a-encontrar" significam:

"Já-encontrados" são constructos mentais criados a partir de experiências anteriores, vivenciadas pelo aluno e que já são parte da imagem de conceito dele e "a-encontrar" são as experiências atuais que interferem no aprendizado anterior podendo modificar a imagem de conceito do aluno. (LIMA, 2007, p. 280).

Em outras palavras, os "já- encontrados" são qualquer experiência anterior a certo aprendizado, ou seja, os pré-requisitos, e que estão presentes na imagem de conceito do indivíduo, e que podem interferir no aprendizado. Já os "a-encontrar" são as experiências atuais de aprendizagem que interfiram no aprendizado de um novo conceito.

Segundo Tall (2004; 2008), à medida que um indivíduo percorre os mundos da matemática ocorrem dificuldades, que exigem experiências anteriores para serem superadas. Porém, cada indivíduo trabalha com estas dificuldades de maneiras diferentes, de acordo com experiências que teve anteriormente e que, por sua vez, podem afetar o aprendizado atual, tanto de maneira positiva quanto negativa. Estas experiências anteriores são os já-encontrados, utilizado por Tall $(2004 ; 2008)$ e que estão presentes na imagem de conceito do indivíduo.

Considera-se que a influência é positiva quando o conceito anterior está bem fundamentado e é coerentemente relacionado com o novo conhecimento, colaborando para que este seja adquirido. Por exemplo, de acordo com Lima (2007), na aritmética um aluno pode já ter conhecimento que 3+4=7 e ao deparar-se com uma nova situação como $3 \mathrm{x}+4 \mathrm{x}$ ele pode evocar o conhecimento anterior e concluir que é igual a $7 \mathrm{x}$. Mas, também, pode influenciar de modo negativo e a autora descreve que o aluno pode deparar-se com a situação 3+4x e também concluir que é igual a 7x o que é um equívoco. Nessa direção, a experiência anterior foi um obstáculo para a aprendizagem. A autora conclui que: “a influência de um já-encontrado pode ser negativa quando não está bem 
estruturado na mente do sujeito e precisa de uma reconstrução, podendo vir a se tornar um obstáculo para uma nova aprendizagem”.

Assim como experiências anteriores, "já-encontrados" podem afetar novos aprendizados, novas experiências também podem interferir em aprendizados anteriores, "a-encontrar". Os "a-encontrar" são experiências que podem ainda não ser parte da imagem do conceito de um indivíduo, mas podem tanto modificá-la quanto vir a fazer parte dela.

Quanto à "definição de conceito", (TALL; VINNER, 1981, p.2), afirmam que é "uma sentença de palavras usadas para descrever um determinado conceito". Segundo os estudiosos, um conceito pode simplesmente ser memorizado pelos estudantes, mas também pode ser aprendido de modo significativo, à medida que tenham oportunidades de criar diferentes imagens conceituais referentes a um determinado conteúdo matemático.

Tall (1992) menciona a dificuldade que os alunos têm de selecionar e usar representações apropriadas. Sobre esse aspecto, ainda que não ofereça uma explicação sobre como cada tipo de dificuldade que se apresenta, Tall comenta textos de outros autores, que consideram serem mais flexíveis os alunos que usam representações simbólicas, numéricas e visuais, mesmo que muitos desses estudantes tenham certa relutância em usar as representações visuais, por estarem acostumados às abordagens numérica e simbólica.

O autor ainda afirma: "eu logo me dei conta de que os gráficos, sozinhos, eram insatisfatórios [...] e vi a necessidade de um movimento mais versátil entre as representações. As gráficas dão insight global qualitativo, enquanto as numéricas dão resultados quantitativos e as simbólicas dão poderosa habilidade manipulativa". (p. 9, grifos do original). Tall conclui que movimentar-se entre as representações é mais importante e cognitivamente mais natural do que focar os três tipos de representações de uma vez só.

Assim, acredita-se que trabalhar um conceito, levando-se em consideração as suas múltiplas representações, é proporcionar aos alunos oportunidades de criar imagens ricas de significados que permitam a compreensão do conceito.

De acordo com Tall (1994), o ensino centrado nos aspectos técnicos e analíticos, que desvaloriza o raciocínio e o uso da representação visual, é uma das razões do insucesso em matemática. Com isso, os estudantes têm dificuldades em fazer a conexão do pensamento visual com o pensamento simbólico e não conseguem passar facilmente 
de uma representação para outra. O autor, ao investigar a compreensão do conceito de derivada, afirma que a visualização gráfica desempenha um papel central na aprendizagem do conceito e na compreensão das relações e propriedades.

Da análise dos resultados das pesquisas aqui citadas, é possível inferir que, em geral, os alunos apresentam dificuldades de abordar o conceito de derivada utilizando diferentes representações, e que essas dificuldades, podem estar relacionadas com a valorização, no trabalho em sala de aula, de aspectos simbólicos em detrimento de aspectos gráficos, o que pode contribuir para a criação de imagens conceituais restritas.

\section{Procedimentos metodológicos}

A fim de atender ao objetivo da pesquisa, foi aplicado um teste formado por seis questões abertas, com o propósito de saber qual o significado atribuído pelos professores à palavra "variação" e à compreensão desse conceito explicitado por diferentes representações. O teste foi respondido individualmente por dez professores de Matemática, alunos de um Curso de Mestrado em Ensino de Matemática, matriculados na disciplina de Fundamentos de Cálculo Diferencial e Integral. Ressalta-se que muitos pesquisadores têm realizado estudos sobre a formação de professores e a construção de conceitos matemáticos, em particular o conceito de derivada, no entanto, as particularidades de estudo de cada grupo, suas experiências pessoais e suas reflexões sobre a prática realizada são diversas e essa diferença contribui para o redimensionamento dos objetivos e das práticas dos professores desses cursos.

A aplicação do teste teve duração de uma hora-aula de 60 minutos. Após a conclusão, as respostas foram separadas e categorizadas. A análise foi elaborada a partir da interpretação feita sobre as informações obtidas nas respostas do teste. De acordo com as questões propostas, foram definidas à priori as seguintes categorias de respostas: a) compreensão da palavra "variação"; b) significado matemático da palavra "variação"; c) relação entre o conceito de taxa de variação e o coeficiente angular da reta tangente e d) significado físico de taxa de variação.

\section{Apresentação e análise dos resultados}

Ao analisar as respostas do teste, optou-se por identificar os dez participantes apenas por letras, para preservar sua identidade. Além disso, não se fez distinção de gênero, chamando cada um de professor. 
São apresentadas, a seguir, cada uma das categorias, as respostas dos professores e as considerações das pesquisadoras. Quando não são citadas outras respostas, isso significa que os demais participantes deixaram em branco a questão.

a) Compreensão da palavra "variação".

Essa primeira categoria está relacionada com as respostas dos professores sobre a primeira questão: o que você pensa quando ouve a palavra "variação"? Conforme as respostas dos professores, diferentes significados são descritos para a palavra.

Professor A- A variação da temperatura hoje foi de 15 a 23 graus.

Professor G- A variação de consumo de combustível por um carro à gasolina é de 10 a 12 litros por quilômetro rodado.

Professor I- A nota máxima do concurso foi 9,0 e a mínima foi 3,5, logo houve uma variação significativa de valores.

Professor J- É algo que não é exato, que varia ou se modifica com o tempo.

Professor K- Os gostos mudam de acordo com a idade.

Observa-se, pelas respostas dos professores, que nenhum deles utilizou uma linguagem matemática ou se referiu ao conceito de taxa de variação. Segundo Tall (1994), isto pode estar ligado ao modo como os conceitos foram abordados em sala de aula na graduação, priorizando-se a linguagem algébrica em detrimento da forma verbal de expressar um conceito.

b) Significado matemático da palavra "variação".

Essa categoria está relacionada à segunda questão do teste: você conhece algum significado matemático da palavra variação?

Como resposta, têm-se as seguintes colocações dos professores:

Professor A- Por exemplo, a variação da distância percorrida por um automóvel num determinado intervalo de tempo.

Professor G- A cesta básica sobe $2 \%$ a cada mês.

Professor I- A temperatura diminui 5 graus a cada 1000 m de altura.

Professor K- O preço é proporcional ao peso do produto.

Professor $\mathrm{H}-A$ variação da variável y em relação à variável $x$, que significa quanto a variável y está mudando, à medida que a variável x muda.

Nessas repostas, pode-se observar que alguns professores conseguem estabelecer relações entre a palavra variação e seu significado matemático, explicitando a taxa de variação e estabelecendo relações com o coeficiente de proporcionalidade. De acordo com Tall e Vinner (1981), os professores expressaram-se segundo as "imagens do 
conceito" de taxa de variação criadas quando este conceito foi construído na graduação. Os demais professores não conseguiram descrever de modo coerente o significado matemático. Pode-se inferir, dessas respostas, que as "imagens do conceito" foram muito pobres ou não foram construídas pelos professores quando o conceito foi abordado pela primeira vez.

Ou seja, pode-se observar que os conhecimentos prévios, isto é, os “Jáencontrados" foram evocados pois alguns professores, não a maioria, conseguiram dar um significado matemático para a palavra variação no sentido matemático, embora não o tenham feito relacionado com o conceito de derivada.

c) Relação entre o conceito de taxa de variação e o coeficiente angular da reta tangente.

A terceira e quarta questões do teste estão relacionadas com essa categoria.

3) Que significado você atribui ao valor de M na expressão abaixo?

$$
\lim _{x \rightarrow x 1}\left(\frac{f(x)-f(x 1)}{x-x 1}\right)=M
$$

4) Observe a figura a seguir e analise se é possível estabelecer uma relação entre a reta que passa pelos pontos $P$ e $Q$ e o significado de $M$ do item anterior.

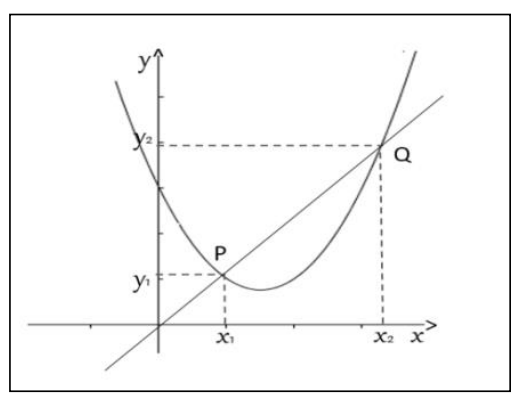

Figura 1: Reta que passa pelos pontos $\mathrm{P}$ e Q

Fonte: os autores

As respostas dos professores às questões mostram que poucos conseguiram estabelecer uma relação entre o valor $\mathrm{M}$, que representa a derivada da função no ponto $\mathrm{x}_{0}$, e o coeficiente angular da reta tangente ao gráfico. Apenas dois deles descreveram que o valor de $\mathrm{M}$ corresponde à derivada da função no ponto $\mathrm{x}_{1}$, e quando o ponto $\mathrm{Q}$ se aproxima do ponto $\mathrm{P}$, o coeficiente angular da reta secante se aproxima do coeficiente angular da reta tangente. Nas suas respostas, colocaram as seguintes explicações:

Professor A- A ideia é a mesma. O valor de $M$ corresponde à derivada da função ou a taxa de variação e, no caso do gráfico, se tomarmos o limite teremos o coeficiente angular da reta tangente. 
Professor G- Se tomarmos os coeficientes angulares das retas secantes, quando o ponto $P$ se aproxima do ponto $Q$ teremos o coeficiente angular da reta tangente que é a taxa de variação.

Professor I - É a taxa de variação de uma função em um ponto, ou seja, é a taxa de variação pontual.

Como somente dois professores conseguiram responder às questões, pode-se inferir que a maioria deles, ao analisar o gráfico, não conseguiu relacionar o coeficiente angular da reta tangente com o conceito de taxa de variação. Também, não conseguiram identificar o mesmo conceito quando é expresso por meio de diferentes representações.

$\mathrm{Na}$ questão proposta o propósito era olhar a mudança do gráfico da reta secante quando muda sua inclinação a partir do deslocamento do ponto $\mathrm{Q}$ para o ponto $\mathrm{P}$. A resposta do professor G está de acordo com as ideias de Gray e Tall (2001), que afirmam que a compreensão do que ocorre com o coeficiente angular da reta secante se dá a partir da análise do gráfico e não a partir de um processo de limite que remete a um tratamento simbólico. Nesse caso a interpretação da taxa de variação se deu a partir da análise da mudança de inclinação da reta secante.

As respostas sugerem a mobilização de elementos da imagem conceitual relativa ao conceito de derivada quando interpretada geometricamente.

d) Significado físico da taxa de variação.

Nessa categoria, foram analisadas as respostas da quinta e da sexta questão.

5) Se um carro percorre $120 \mathrm{~km}$ de uma estrada reta em $3 \mathrm{~h}$, qual é sua velocidade média? Você consegue estabelecer uma relação entre a terceira e quarta questão e o conceito de velocidade média?

6) O quadro a seguir mostra as coordenadas de uma partícula movendo-se ao longo de um eixo durante o intervalo de tempo $t=4$ a $t=6$ minutos, em que $s$ indica a distância percorrida.

\begin{tabular}{|c|c|c|c|c|c|c|c|c|c|}
\hline $\mathrm{t}$ (minutos) & 4,00 & 4,50 & 5,00 & 5,50 & 5,80 & 5,90 & 5,95 & 5,98 & 6,00 \\
\hline $\begin{array}{c}\mathrm{s} \\
\text { (metros) }\end{array}$ & 1,00 & 1,25 & 2,00 & 3,25 & 4,24 & 4,61 & 4,80 & 4,92 & 5,00 \\
\hline
\end{tabular}

Quadro 1: Coordenadas de uma partícula

Fonte: os autores

Qual a velocidade média nos intervalos [4,00, 6,00], [4,50, 6,00], [5,00, 6,00], $[5,50,6,00],[5,80,6,00],[5,90,6,00],[5,95,6,00],[5,98,6,00]$ ? Qual é a velocidade no instante $t=6 \mathrm{~min}$ ?

Para essas questões, têm-se as seguintes respostas: 
Professor A- O carro percorreu $120 \mathrm{~km}$ em 3h, mas essa velocidade não pode ter sido a mesma em todo percurso. Sabendo o percurso e o tempo, pode-se obter uma média, nesse caso, será de 40km/h. Na última questão, é necessário calcular a velocidade média em cada intervalo. Para calcular a velocidade média, preciso calcular os valores dos quocientes $\frac{S-S 0}{T-T 0}$ para cada intervalo. Nas minhas contas, obtive os valores, $2 ; 2,5 ; 3 ; 3,5 ; 3,8 ; 3,9 ; 4 ; 4$. A conclusão que eu chego é que no instante $t=6$ min a velocidade é $4 \mathrm{~km} / \mathrm{h}$.

Professor C- A velocidade média não se calcula do mesmo modo que a velocidade instantânea. Na primeira pergunta, a velocidade média é de $40 \mathrm{~km} / \mathrm{h}$, mas o carro não anda todo percurso com essa velocidade constante. Na segunda pergunta, preciso fazer os cálculos

$$
\begin{gathered}
\frac{5-1}{6-4}=2 ; \frac{5-1,25}{6-4,5}=2,5 ; \quad \frac{5-2}{6-5}=3 ; \frac{5-3,25}{6-5,50}=3,5 \\
\frac{5-4,24}{6-5,80}=3,8 ; \quad \frac{5-4,61}{6-5,90}=3,9 ; \frac{5-4,80}{6-5,95}=4 ; \frac{5-4,92}{6-5,98}=4 .
\end{gathered}
$$

A velocidade no instante $6 \mathrm{~min}$ é $4 \mathrm{~km} / \mathrm{h}$, pois quanto mais próximo do instante 6min, o valor ficou constante e igual a $4 \mathrm{~km} / \mathrm{h}$.

Professor G- No primeiro caso, a velocidade média é $40 \mathrm{~km} / \mathrm{h}$. Na segunda pergunta, precisa calcular a velocidade média em cada intervalo.

O Professor G fez os mesmos cálculos do Professor C e obteve o valor de 4km/h.

Os demais professores responderam somente à primeira pergunta obtendo o mesmo valor de $40 \mathrm{~km} / \mathrm{h}$.

Quanto à segunda pergunta, o Professor G respondeu: A relação que eu vejo com a terceira e quarta questão é que a velocidade média está relacionada com o coeficiente angular da reta secante, e a velocidade instantânea está relacionada com o coeficiente angular da reta tangente.

Portanto, observou-se que o Professor G conseguiu estabelecer relações entre as diferentes representações do conceito de taxa de variação. Pode-se inferir que este professor criou imagens significativas do conceito quando este conhecimento foi construído na graduação, e fez uso delas para analisar e interpretar as questões. Os demais professores tiveram dificuldades para responder e não conseguiram estabelecer relações entre as diferentes representações. 
Pinto (2006) refere-se ao fato de que as atividades matemáticas, na visão de Tall, são muito sustentadas pelo uso de certas palavras da língua materna- tais como "limite" que têm um significado matemático específico. Já a visualização é uma "representação intermediária que enriquece a experiência matemática com exploração concreta adicional do objeto" (PINTO, 2006, p.200). Nessa questão, a visualização do gráfico da função e da reta secante foi de fundamental importância para compreensão do conceito de Taxa de Variação e sua relação com outras representações do mesmo conceito.

\section{Considerações finais}

Nos cursos de Licenciatura em Matemática, são trabalhados os conceitos fundamentais do Cálculo Diferencial e Integral. Os alunos, em geral, estudam o conceito de taxa de variação durante sua graduação, mas os resultados desta pesquisa mostram que esses participantes criaram imagens do conceito muito restritas, que pouco contribuíram para a compreensão do conceito. Da análise das respostas às questões propostas, o estudo revelou que a maioria desses alunos possui problemas em termos de conhecimentos do conceito de taxa de variação, pois não conseguiu justificar, de forma conveniente, as questões do teste, bem como não conseguiu estabelecer relações entre as diferentes representações do conceito.

Em relação ao propósito desta pesquisa, constatou-se que os professores recordaram alguns conceitos relacionados à taxa de variação que foram, provavelmente, memorizados ao longo da formação inicial, mas sentiram dificuldades de aplicá-los em um novo contexto de conhecimento. No que se refere à relação entre as diferentes formas de representar o conceito, ficou clara a dificuldade que tiveram para identificar e justificar matematicamente. Outras dificuldades foram observadas em relação à compreensão do conceito de taxa de variação, oriundas, possivelmente, da forma errônea de entendê-lo e devido a equívocos relacionados com a falta de compreensão e a criação de poucas imagens do conceito.

As dificuldades de interpretar o conceito de taxa de variação detectadas nesta pesquisa foram aspectos que se buscou aprofundar ao longo da disciplina de Fundamentos de Cálculo Diferencial. O conceito de taxa de variação é fundamental na Matemática e sua compreensão tem implicações na resolução de problemas em níveis avançados. Assim, o conhecimento que os alunos têm sobre o conceito e a exploração de 
suas diferentes representações e suas relações precisam ser trabalhadas nas disciplinas de Cálculo.

Dos resultados da análise dos dados da pesquisa, emergem questões que merecem um aprofundamento, tais como: qual a influência desta abordagem na compreensão dos conceitos de taxa de variação, de derivada e suas diferentes interpretações pelos alunos? Os alunos, ao chegarem à universidade trazem características dos Mundos Conceitual Corporificado e Axiomático Formal em sua apropriação do conceito ou apenas trabalham com o mundo operacional simbólico, desenvolvendo a resolução de exercícios simples, pautados na mera reprodução do modelo de resolução?

A essência do conceito de derivada está ligada ao conceito de taxa de variação e sua relação com o coeficiente angular de uma reta tangente e da velocidade média e instantânea de uma partícula em movimento. Muitos dos alunos, após a realização das atividades, evocaram os conhecimentos de derivada e sua aplicação em problemas de máximo e mínimo em diversas áreas. Após as discussões que se estabeleceram foi possível verificar como os "já-encontrados" influenciaram a reconfiguração das imagens conceituais que os alunos possuíam sobre o conceito de derivada.

De acordo com Lima (2007), ao observarmos os conceitos "já-encontrados" e os "a-encontrar" a partir da análise dos dados, é possível identificar as experiências que esses alunos tiveram, ou que estão tendo, que interferem no trabalho deles e revelam também, como podem ser as imagens de conceito dos alunos. Esses aspectos são importantes para os professores que atuam em cursos de formação continuada pois, é possível identificar as experiências que ainda precisam ser estruturadas nas imagens de conceitos dos alunos e planejar atividades que propiciem uma compreensão significativa desses conceitos.

O trabalharem, na sala de aula, diferentes conceitos da matemática, muitos professores esperam que a imagem conceitual seja formada ou controlada pela definição formal. Todavia, de acordo com Vinner (1989), a definição não tem poder cognitivo sobre o pensamento conceitual do aluno. É importante notar que a reconstrução de uma definição formal nem sempre indica um entendimento do conceito, visto que tal definição pode ter sido adquirida por memorização.

Percebemos, por meio da ideia de imagem conceitual e definição conceitual, que a aprendizagem de conceitos, analisada por meio do conceito de taxa de variação, não está bem consolidada. Certamente, o ensino de conceitos precisa ser revisto e aperfeiçoado. É necessário investigarmos formas de ensinar conceitos aos alunos, de modo a permitir que 
estes formem tanto a imagem quanto a definição conceitual de maneira satisfatória. É necessário focar menos na resolução de exercícios e aprendizagem mecânica e mais no conceito em si e na sua aplicação e criar condições, na sala de aula, para que experiências neste sentido aconteçam.

As preocupações com a aquisição de novos conceitos do Cálculo Diferencial e Integral são pertinentes porque pretende-se compreender como se dá a aprendizagem dos conceitos desta disciplina e, assim, um aprofundamento nestas discussões pode contribuir para que a aprendizagem, de fato, ocorra. As conclusões deste trabalho apontam a necessidade de, não apenas em relação ao conceito de taxa de variação, mas também em relação a outros conceitos, se valorizar e integrar, nas práticas de sala de aula, representações diversificadas, no sentido de criar imagens conceituais que deem significado aos tópicos abordados.

\section{Referências}

ALMEIDA, M. V.; IGLIORI, S. B. C. Educação Matemática no Ensino Superior e abordagens de Tall sobre o ensino/aprendizagem do Cálculo. Educação Matemática Pesquisa, São Paulo, v.15, n.3, p.718-734. 2013.

BRASIL. Ministério da Educação. Conselho Nacional de Educação. Diretrizes Curriculares Nacionais para os Cursos de Matemática, Bacharelado e Licenciatura. 2001. Disponível em: <http:// www.mec.gov.br>. Acesso em: 15 de mar. 2015.

FROTA, M. C. R.; NASSER, L. (Orgs.). Educação Matemática no Ensino Superior: pesquisas e debates. Recife. SBEM, 2009.

FROTA, M. C. R.; BIANCHINI, B. L.; CARVALHO, A. M. F. T. Marcas da Educação Matemática no Ensino Superior. (Orgs.). Campinas: Papirus, 2013.

GRAY, E.; TALL, D. Relationships between embodied objects and symbolic procepts: an explanatory theory of success and failure in mathematics. In: PSYCHOLOGY OF

MATHEMATICS EDUCATION, 25., 2001, Utrecht. Proceedings... Utrecht, 2001. p. 65-72.

LIMA, R. N. de. Equações Algébricas no Ensino Médio: uma jornada por diferentes mundos da Matemática. 2007. Tese (Doutorado em Educação Matemática) - Pontifícia Universidade Católica de São Paulo, 2007.

MEYER, C. Derivada/Reta Tangente: imagem conceitual e definição conceitual. 2003. Dissertação (Mestrado em Educação Matemática) - Pontifícia Universidade Católica de São Paulo, São Paulo, 2003.

MEYER, C.; IGLIORI, S.B.C. Um estudo sobre a interpretação geométrica do conceito de derivada por estudantes universitários. In: SEMINÁRIO INTERNACIONAL DE PESQUISA EM EDUCAÇÃO MATEMÁTICA, 2., 2003, Santos. Anais... São Paulo: SBEM, 2003. 
PINTO, M.M.F.; CUNHA, S.R. O conhecimento esperado sobre limites e continuidade a partir de uma análise das provas unificadas de Cálculo I na UFRJ. Educação Matemática Pesquisa, São Paulo, v. 16, n. 1, p. 259-278, 2014.

PINTO, M. M. F. Learning and Experiencing the Mathematical Enterprise. In: SIMPSON, A. (Ed.). Retirement as Process and Concept: A Festschrift for Eddie Gray andDavid Tall. Praga: KarlovaUniversita, 2006. p. 195-204.

TALL, D. Students' difficulties in Calculus. In: INTERNATIONAL CONGRESS ON MATHEMATICAL EDUCATION, 7., 1992, Québec. Anais... Québec,1992. Disponível em: <http://citeseerx.ist.psu.edu/viewdoc/download?doi=10.1.1.377.2989\&rep=rep1\&type=pdf > . Acesso em: 03 jun.2017.

TALL, D. Computer environments for the learning of mathematics. In: BICHLER, R. et al. (Eds.) Didatics of mathematicsas a scientific discipline. Dordrecht: Kluwer, 1994. p. 189-199.

TALL, D.; VINNER, S. Concept image and concept definition in mathematics with particular reference to limit and continuity. Educational Studies in Mathematics, [s.1.], v.12, p. 151-169, 1981.

TALL, D. Thinking through three worlds of mathematics. In: INTERNATIONAL CONFERENCE FOR THE PSYCHOLOGY OF MATHEMATICS EDUCATION, 28., 2004, Bergen, Norway. Proceedings... Bergen: PME, 2004. p. 281-288.

TALL, D. The Transition to Formal Thinking in Mathematics. Mathematics Education Research Journal, v. 20, n. 2, p. 5-24, 2008.

TALL, D. How humans learn to think mathematically. Cambridge: Cambridge University Press, 2013.

VINNER, S. The avoidance of visual consideration in Calculus students. Focus on Learning Problems in Mathematics, v.2, n. 11, p.149-156, 1989.

VRANCKEN, S.; ENGLER, A. Una Introdución a la Derivada desde la Variación y el Cambio: resultados de una investigación con estudiantes de primer año de la universidad. Bolema, Rio Claro, v.28, n.48, p.449-468, 2014.

Recebido em: 23 de fevereiro de 2018.

Aceito em: 29 de abril de 2018. 